\title{
Comparative analysis of oocyte transcript profiles reveals a high degree of conservation among species
}

\author{
Maud Vallée, Kazuhiro Aiba ${ }^{1}$, Yulan Piao ${ }^{1}$, Marie-France Palin ${ }^{2}$, Minoru S H Ko ${ }^{1}$ and \\ Marc-André Sirard
}

Département des Sciences Animales, Centre de Recherche en Biologie de la Reproduction (CRBR), Université Laval, Québec, Québec, G1K 7P4 Canada, ${ }^{1}$ Developmental Genomics and Aging Section, Laboratory of Genetics, National Institute on Aging (NIA), National Institutes of Health (NIH), Baltimore, Maryland 21224, USA and ${ }^{2}$ Dairy and Swine Research and Development Centre, Agriculture and Agri-Food Canada, Lennoxville, Québec, J1M 1Z3 Canada

Correspondence should be addressed to M-A Sirard; Email: marc-andre.sirard@crbr.ulaval.ca

\begin{abstract}
Cross-species comparison of gene expression is a powerful approach for discovering genes that have been conserved throughout evolution. Conserved genes are presumably very important in the mechanisms related to the unique molecular functions in oocytes. The objective of this study was to identify genes expressed in the oocyte and conserved across three diverse vertebrate species. We report the global gene expression profiles of Bos taurus and Xenopus laevis oocytes on an NIA mouse development microarray that consists of 60-mer oligonucleotide probes representing more than 20000 mouse transcripts derived from stem cell, oocyte, and early embryo cDNA libraries. Analysis based on intensity values revealed that 9853 and 10046 genes are expressed in bovine and Xenopus oocytes respectively. Furthermore, previously published microarray data on preimplantation development in the mouse were used for a comparative analysis of global oocyte gene expression profiles. Interestingly, a substantial proportion of the genes expressed in mouse oocytes is conserved between the three species $(74 \%, 7275$ genes). Moreover, functional annotation of these conserved oocyteexpressed genes confirmed that certain functions are conserved among the three species. RNA metabolism and cell cycle were among the over-represented Gene Ontology terms in the biological process category. Finally, a pattern-matching analysis identified 208 conserved maternally expressed genes. Results from these cross-species hybridizations allowed numerous genes expressed in oocytes and conserved between Mus musculus, $B$. taurus, and $X$. laevis to be identified. This comparative analysis of oocyte transcript profiles revealed a high degree of conservation among species.

Reproduction (2008) 135 439-448
\end{abstract}

\section{Introduction}

In vertebrates, early embryonic development is supported by maternal mRNAs and proteins synthesized and stored during oogenesis (Rodman \& Bachvarova 1976). These maternal factors are critical for development between the fertilization and the maternal-embryonic transition, when transcription of the embryonic genome becomes fully activated. Recent gene-targeting studies have revealed essential developmental roles for oocytespecific genes: specifically, Gdf9 (Dong et al. 1996), Bmp15 (Yan et al. 2001), Zp1, Zp2, Zp3 (Rankin et al. 1996, 1999, 2001), Mater (Tong et al. 2000), Figla (Soyal et al. 2000), Npm2 (Burns et al. 2003) and Zar1 (Wu et al. 2003), which are all essential for supporting oogenesis, fertilization, or proper early embryonic development prior to embryonic genome activation.

Previous studies have revealed that $\sim 15700$ genes are involved in mouse embryonic development (Stanton et al. 2003) and that 196 of them are uniquely expressed in unfertilized and fertilized eggs (Sharov et al. 2003). There is also evidence that genes expressed in oocytes and early embryos are conserved among species, since numerous orthologs have been found in mice and humans by in silico analysis (Stanton \& Green 2001, 2002). However, our understanding of the oocyte transcriptome and the identity of key oocyte-expressed genes is far from complete. Furthermore, characterization of gene expression in oocytes is necessary and will provide additional insight into the regulation of oocyte maturation, fertility, and preimplantation development.

A variety of approaches have been used to study gene expression in oocytes. RT-PCR is a method of choice for profiling expression of known genes of interest on an individual basis. However, the growing need for genome-wide analysis has motivated the development of high-throughput techniques such as the analysis of expressed sequence tags (EST; Adams et al. 1991), differential display RT-PCR (Liang \& Pardee 1992), 
SAGE analysis (Velculescu et al. 1995), and suppressive subtractive hybridization (Diatchenko et al. 1996). These techniques have been successfully used during the past few years to identify important genes in oocytes (Ko et al. 2000, Neilson et al. 2000, Robert et al. 2000, Monk et al. 2001, Sharov et al. 2003, Zeng \& Schultz 2003, Pennetier et al. 2005, Vallee et al. 2005).

Although these approaches have shed some light on the molecular basis of preimplantation development, each has major limitations. Microarray analysis has proved to be the most powerful approach for global gene expression profiling (Schena et al. 1995). The development of DNA microarray technology has permitted thousands of genes to be analyzed simultaneously, and the technology has become increasingly popular as a means of identifying differentially expressed genes in complex biological models. Furthermore, a linear amplification method utilizing an in vitro transcription reaction is now being widely used for microarray experiments (Eberwine 1996). This approach is particularly interesting since it circumvents many of the problems inherent with the most commonly employed amplification strategies that depend on PCR. Thus, the ability to amplify the small amounts of mRNA present in oocytes, which can only be isolated in limited quantities, makes it feasible to generate enough material for microarray analysis. Numerous studies have been performed to analyze oocytes global gene expression profiling in different species such as mouse, cow, Xenopus, rhesus monkey, and human using a variety of microarray platforms (Altmann et al. 2001, Bermudez et al. 2004, Dobson et al. 2004, Hamatani et al. 2004, Wang et al. 2004, Yao et al. 2004, Zeng et al. 2004, Arraztoa et al. 2005, Misirlioglu et al. 2006, Fair et al. 2007, Gasca et al. 2007, Ghanem et al. 2007, Patel et al. 2007, Steuerwald et al. 2007, Su et al. 2007). Another approach to microarray-based research is the use of cross-species hybridizations for comparative analysis to reveal evolutionarily conserved mechanisms and pathways. Several studies have performed different analyses to validate the accuracy and precision of results obtained through microarray cross-species hybridization (Chismar et al. 2002, Ji et al. 2004, Renn et al. 2004, Bar-Or et al. 2006). Also, different microarray platforms have been used for cross-species hybridization: custom oligo arrays (Chalmers et al. 2005), Affymetrix arrays (Shah et al. 2004, Grigoryev et al. 2005), cDNA microarray (Adjaye et al. 2004, 2007, Donaldson et al. 2005), and cDNA macroarray (Robert et al. 2002, Dalbies-Tran \& Mermillod 2003). Biologically meaningful information has been obtained from all these studies.

We have previously shown that cross-species hybridizations using cDNA microarrays give very specific results and are very valuable for oocyte gene expression profiling (Vallee et al. 2006). We have also shown that many of the genes expressed in oocytes are conserved between mouse, cow, and Xenopus (Vallee et al. 2006). In the present study, we have performed cross-species hybridizations of Bos taurus and Xenopus laevis oocytes on a microarray platform containing 60-mer oligonucleotide probes representing more than 20000 unique mouse transcripts, assembled primarily from sequences of stem cell, oocyte, and embryo cDNA libraries (Carter et al. 2003). We consider this microarray platform to be best suited for comparative analysis of the oocyte transcriptome since it should contain most of the genes expressed in oocytes, including low copy-number genes. Previously published microarray data obtained from a study on mouse preimplantation development, using the same platform (Hamatani et al. 2004), were also used in the present analysis. This comparative analysis revealed a high degree of conservation among maternal transcripts expressed in Mus musculus, B. taurus, and X. laevis oocytes. Working simultaneously with three distantly related species should help us better to understand the oocyte transcriptome and the identity of key oocyteexpressed genes with functions in important evolutionarily conserved mechanisms.

\section{Results}

\section{Microarray experimental design}

The NIA mouse development microarray used in this study consists of more than 20000 mouse gene features derived from stem cell, oocyte, and early embryo cDNA libraries (Carter et al. 2003). We will use the term 'gene' instead of gene feature in descriptions of microarray data. Bovine and Xenopus oocyte cRNA were used as probes to perform three replicates of cross-species hybridizations on a mouse development microarray. A universal mouse reference RNA (Stratagene, La Jolla, CA, USA) was used for all hybridizations, so that all developmental stages could be compared (Weil et al. 2002). In addition, microarray data from mouse preimplantation development derived from a previous study by Hamatani et al. (2004) were used to perform a comparative analysis of global oocyte gene expression profiles and a patternmatching analysis for identifying conserved maternally expressed genes.

\section{Reproducibility of cross-species hybridizations}

For each experiment, reproducibility was verified by comparing the logarithmic signal intensity values of all microarray spots between three replicated experiments. The correlation coefficients for the signal intensities of all microarray spots between these replicates were calculated in a pair-wise manner using the NIA Array Analysis tool (Sharov et al. 2005a). The results show that for our cross-species hybridizations the correlation is relatively 
Table 1 Correlation coefficients of cross-species hybridization replicate experiments ${ }^{a}$.

\begin{tabular}{lcll}
\hline & Rep1 & Rep2 & Rep3 \\
\hline Bovine & & & \\
Rep1 & 1 & 0.91 & 0.90 \\
Rep2 & & 1 & 0.95 \\
Rep3 & & & 1 \\
Xenopus laevis & 1 & 0.91 & 0.90 \\
Rep1 & & 1 & 0.93 \\
Rep2 & & & 1 \\
Rep3 & & & \\
\hline
\end{tabular}

${ }^{\text {aPearson }}$ correlation for each microarray spots comparing replicate experiments. Correlations were determined using log base(2)

fluorescence intensities of the data from every microarray spot.

high between replicates for bovine (0.90-0.95) and Xenopus (0.90-0.93; Table 1). These correlation coefficients are comparable with those obtained from samespecies hybridizations using mouse oocytes (0.94-0.96; Hamatani et al. 2004). Next, an unsupervised hierarchical clustering using all the replicates was performed using the NIA Array Analysis tool (NIA, Baltimore, MD, USA; Sharov et al. 2005a). This procedure independently clustered all the replicates by their appropriate species with minimal branch tree distances between species (Fig. 1).

\section{Global analysis of gene expression in bovine and Xenopus oocytes}

Data from the cross-species microarray hybridizations were uploaded in the NIA Array Analysis tool and a threshold was established to determine which genes were considered as expressed. This threshold was set according to the plot of error function (s.D. (= square root of the error variance) versus expression level (Log intensity)) to ensure minimal s.D. Genes with a mean log signal intensity above the established threshold ( $\log 3.0)$ were considered to be expressed, whereas genes with signal intensities below the threshold were designated 'absent'. This analysis, based on the intensity values, revealed that the number of genes detected in bovine and $X$. laevis oocytes is fairly similar: 9853 and 10046 genes are expressed in bovine and Xenopus

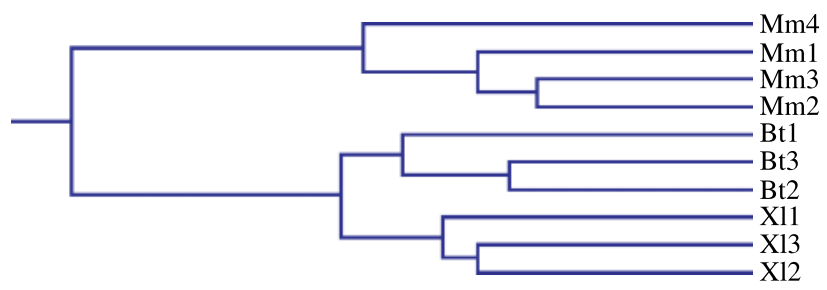

Figure 1 Hierarchical clustering of replicates. The dendogram shows that all replicates are clustered by their appropriate species $(n=3$ for bovine and Xenopus, $n=4$ for mouse). Mouse microarray data are derived from Hamatani et al. (2004). Mm, Mus musculus; Bt, Bos taurus; XI, Xenopus laevis.
Table 2 Genes detected in oocytes ${ }^{\mathrm{a}}$.

\begin{tabular}{lccc}
\hline & Bovine & Xenopus & $\begin{array}{c}\text { Common } \\
\text { in three } \\
\text { species }^{\text {b }}\end{array}$ \\
\hline Genes expressed in oocytes & 9853 & 10046 & $7275(74 \%)$ \\
Average log signal intensity & 3.47 & 3.45 & 3.55 \\
Oocyte-specific genes $^{\mathrm{c}}$ & 90 & 92 & $82(63 \%)$ \\
Average log intensity & 3.42 & 3.46 & 3.65 \\
\hline
\end{tabular}

${ }^{\mathrm{a} G e n e s}$ with a mean log signal intensity above threshold (log $\left.>3.0\right)$.

${ }^{\mathrm{b}}$ The proportion of genes common to the three species relative to the number of genes expressed in mouse oocytes is given in (\%). ${ }^{\mathrm{c}}$ Genes recovered only from mouse unfertilized and fertilized egg cDNA libraries and not present in other analyzed tissues (Sharov et al. 2003).

oocytes respectively (Table 2 ). The maximum average log signal intensity observed is 3.47 and 3.45 in bovine and Xenopus oocytes respectively (Table 2 ).

\section{Comparative analyses of genes expressed in mouse, bovine, and Xenopus oocytes}

A substantial proportion of genes expressed in mouse oocytes are common to the three species $(74 \%, 7275$ genes; Table 2, Fig. 2). Furthermore, genes expressed in oocytes show a wide range of signal intensities from faint to high expression for the three species (Fig. 2). Interestingly, the signal intensities in the three species were also similar (Fig. 2). Those results suggest that genes conserved in oocytes of all three species also have similar expression levels. Genes with low expression levels in the mouse oocytes also have low expression levels in bovine and Xenopus oocytes, and the same applies to highly expressed genes. The species distribution of oocyte-expressed genes is presented as a Venn diagram according to their detection in oocytes of one, two, or three species (Fig. 3). Over 1000 genes appear to be expressed only in mouse oocytes, suggesting either a lack of homology between the species for these specific oligonucleotides and/or genes or possibly an actual interspecific difference in oocyte gene expression. Detection of genes expressed only in B. taurus, $X$. laevis, and $M$. musculus could reflect real differences in oocyte physiology between species.

To characterize further the subpopulation of genes conserved in all three species, we used the large preimplantation EST collections in which 196 genes recovered only from unfertilized and fertilized mouse egg cDNA libraries, and not present in other tissues analyzed, were identified (Sharov et al. 2003). Of these 196 genes, 154 were on the NIA Mouse 22K Microarray and 134 showed statistically significant expression changes during preimplantation stages (Hamatani et al. 2004). Among those genes, 82 were found to be expressed in oocytes of all three species (Table 2). As previously mentioned, these oocyte-specific genes also showed a wide range of signal intensities from faintly expressed to highly expressed (Fig. 2). 

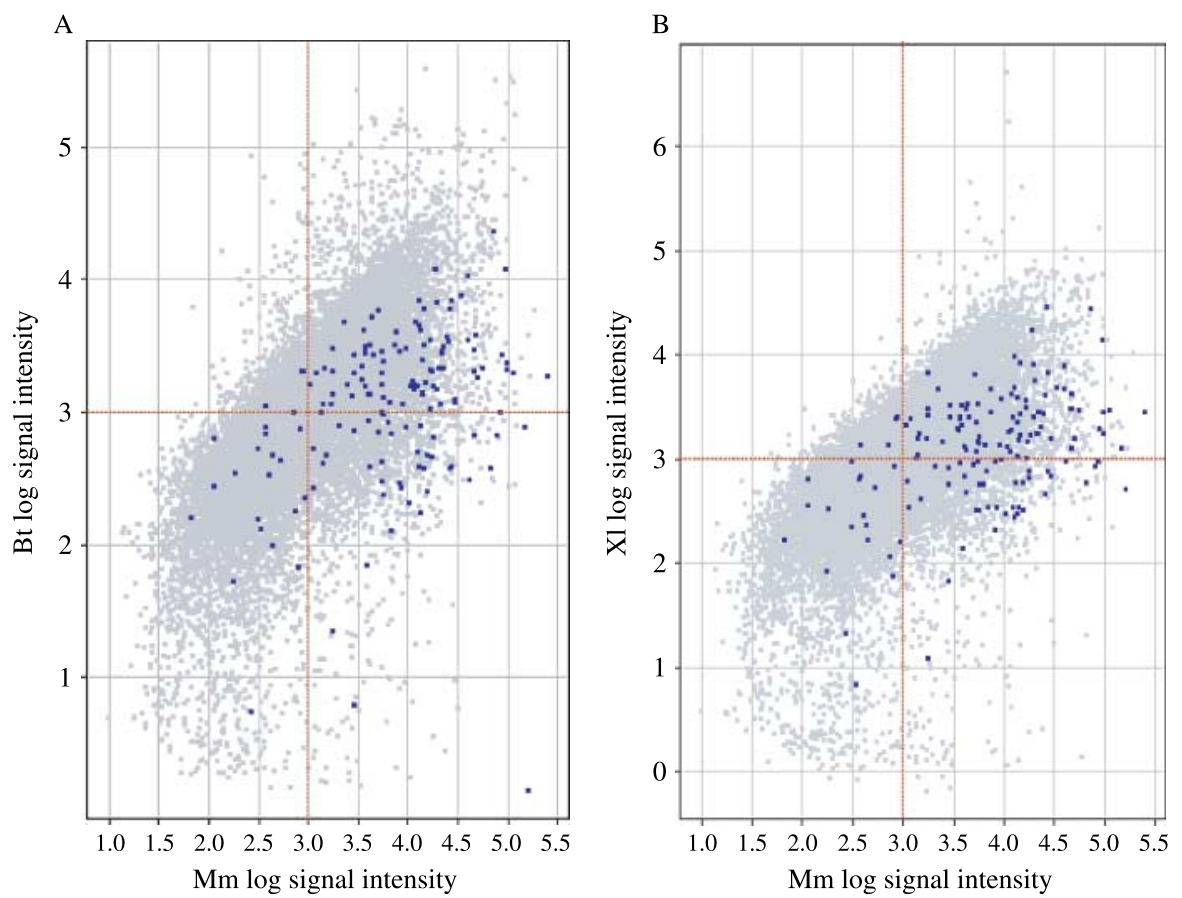

Figure 2 Scatter plot of mean log signal intensities of oocyte-specific genes conserved among species. Mean log signal intensity for (A) bovine and (B) Xenopus laevis oocytes plotted against mean log signal intensity for mouse oocytes. Gray spots represent all the genes on the microarray and blue spots represent oocyte-specific genes (82/154). The red dotted line corresponds to the threshold value (log 3.0). Mm, Mus musculus; Bt, Bos taurus; $\mathrm{XI}$, Xenopus laevis.

\section{GO terms associated with oocyte-expressed genes conserved in all three species}

To find the Gene Ontology (GO) terms associated with the 7275 conserved genes expressed in oocytes, we used the tool in the NIA Mouse Gene Index 8.0 (Sharov et al. 2005b). The NIA Mouse Gene Index 8.0 contains all transcripts found in the microarray platform used in this study. This tool associates the proper GO terms from the Gene Ontology Consortium annotation categories (biological processes, cellular components, and molecular functions) for each gene and generates a list of over-represented GO terms in our population (Ashburner et al. 2000). From this analysis, several over-represented categories were identified from our candidate genes. RNA metabolism, ribonucleoprotein complex, and RNA binding were the most over-represented GO terms in conserved oocyte-expressed genes for the biological process, cellular component, and molecular function categories respectively (Fig. 4). Example of genes in these overrepresented categories that are conserved in oocytes are: Cpeb1, Eif4e2, Gemin6, Pabpn1, Paip1, Pum1, Rnpc2, Slbp, and Snrpd2. Cell cycle and cellular division were also among the biological processes over-represented in the genes conserved in oocytes. Bub1 and Mphosph6, known for their association with the cell cycle and/or cell division and previously identified as oocyte specific, were found in the present study to be associated with this category.

\section{Conserved maternally expressed genes in development}

Finally, to examine further the possible roles of genes according to their expression patterns during embryonic development, the conserved oocyte-expressed gene list was used for pattern-matching analysis. The spindlin (Spin) gene was used as template to identify conserved maternal genes. The results of this pattern-matching analysis for conserved oocyte-expressed genes revealed that 208 genes display the characteristics of conserved maternally expressed genes in the mouse (Fig. 5A). The list of known genes is presented in Fig. 5B. Among those

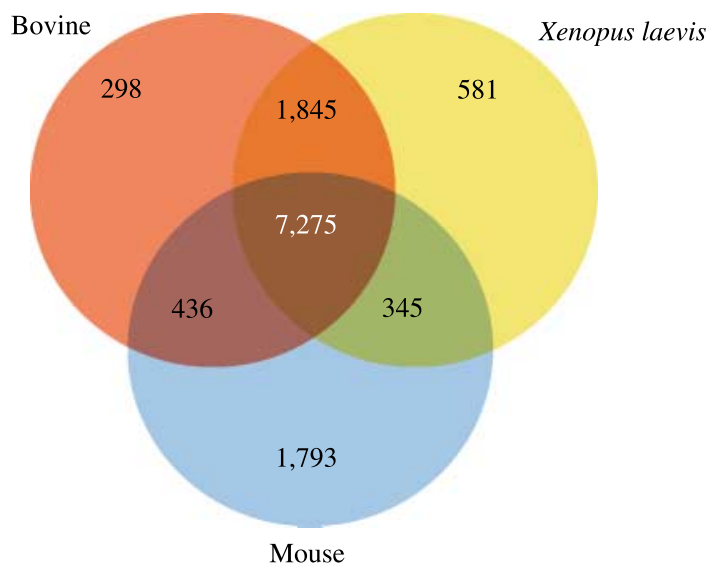

Figure 3 Venn diagram representing clones present in oocytes of one, two, or all three species. Transcripts are detected when their mean log signal intensities are above the threshold $(>\log 3.0)$. 
A

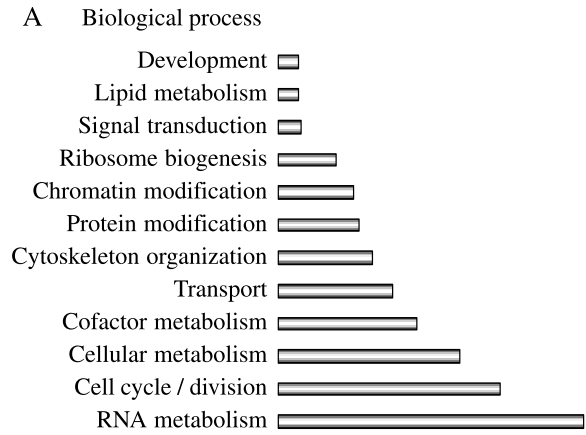

B Cellular component

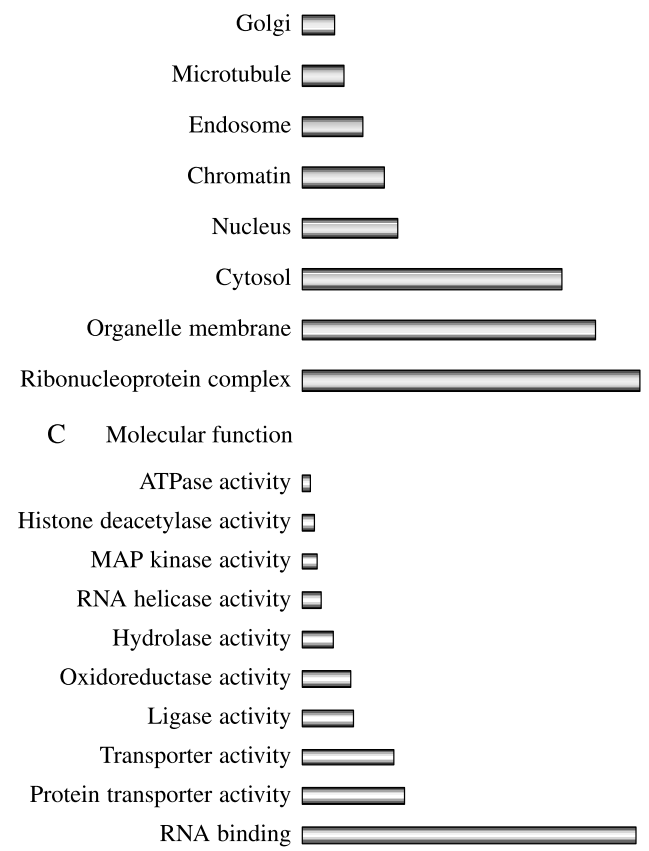

Figure 4 Selected GO categories over-represented in conserved oocyteexpressed genes. GO categories associated with conserved genes expressed in oocytes were identified using the NIA Mouse Gene Index 8.0 tool. Graphic representation of the GO term frequencies associated with (A) biological process, (B) cellular component, and (C) molecular function categories.

identified, some were previously known as maternal genes, such as Bcl2/10, Gdf9, Tcl1 and Zar1. More interestingly, this analysis also identified uncharacterized transcripts such as D6Ertd474e, BC066140, 2610019P18Rik, and C230081A13Rik. These maternally expressed genes are particularly interesting, mainly because of their expression patterns during embryonic development but also because they are conserved between the three species.

\section{Discussion}

The main objective of this study was to identify conserved oocyte-expressed genes. We believe that a comparative analysis performed on three distantly related species is a powerful tool for identifying key
A

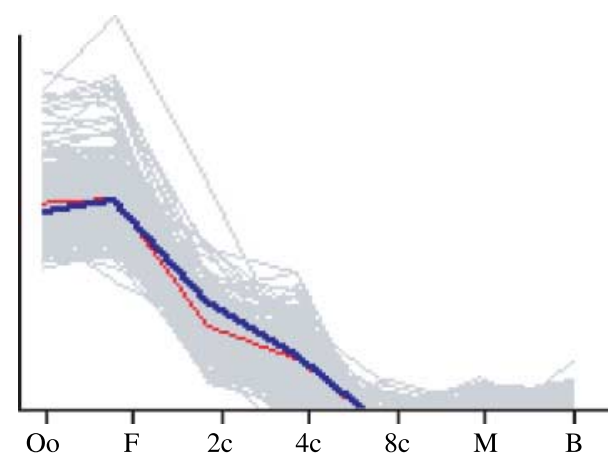

B

\begin{tabular}{l}
\hline Gene symbols \\
\hline Acbd3; Akap10; Arl6; Arntl; Bcl2110; Bfar; Birc3; Bmi1; \\
Commd3; Brd1; Camsap1; Catnbip1; Cdc3711; Cdc42se2; \\
Cetn4; Clk1; Clock; Cobll1; Cpa1; Csad; Csnk1g3; Ddhd1; \\
Dnajb4; Dpys13; E2f5; Eif2ak4; Eppb9; Ezh2; Fbxo28; \\
Fbxw4; Fchsd2; Gbas; Gdf9; Glcci1; Gna12; Gpr125; \\
Gprasp1; Gyg1; Hip2; Hrmt111; Ica1; Ipp; Klhdc2; Lrp6; \\
Mad211; Mak3; Mapkbp1; Mb12; Mbtd1; Mdm4; Misc12; \\
Mitc1; Mllt10; Moap1; Itpk1; Mpdz; Mphosph6; Mycbp; \\
Rragc; Nab1; Nalp4a; Obox1; Osp94; Papss1; Pdpk1; \\
Pex13; Phc2; Plag1; Plk4; Pnrc1; Pols;Ppp4r2; Prg1; \\
Prkcm; Ptp4a2; Q7tpx9; Rab14; Rasa2; Rb1; Rhced; \\
Rhpn2; Ripk5; Rnf141; Rnf34; Sestd1; Sfmbt1; Sh3d1b; \\
Siah2; Sin3a; Slbp; Slco3a1; Smarca2; Smoc2; Snx9; Spin; \\
Spire1; Stard3nl; Syt14; Taf4a; Tank; Tcf20; Tcfap2e; Tcl1; \\
Tgfb2; Tiam2; Tusc3; Ube2d3; Unc13b; Usp31; Usp52; \\
Vamp4; Vil1; Wdr42a; Ythdf3; Zar1; Zbed3; Zc3hdc1; \\
Zcchc2; Zfyve21
\end{tabular}

Figure 5 Identification of conserved maternally expressed genes through pattern matching. The analysis was performed using the NIA microarray analysis tool with Spindlin gene (Z00008605-1) as template (tenfold change threshold and 0.90 correlation threshold). (A) Expression profiles of 208 genes identified through pattern matching. The $y$-axis indicates the relative expression levels of each gene and the $x$-axis indicates each developmental stage. The bold black line shows the pattern of the template gene (Spin), gray lines are centered gene intensities, and the red line is the average intensity of the genes found. Oo, oocyte; $F$, fertilized egg; 2c, 2-cell; 4c, 4-cell; 8c, 8-cell; M, morula; B, blastocyst. (B) List of known genes identified through this pattern-matching analysis.

oocyte-expressed genes with important functions in evolutionarily conserved mechanisms and should provide a better understanding of the oocyte transcriptome.

An essential criterion for the application of crossspecies experiments is data reproducibility. The comparison of correlation coefficients between samespecies and cross-species hybridizations revealed that the latter were sufficiently high to confirm acceptable reproducibility between experimental replicates. However, the cluster dendogram revealed a crossspecies effect. The sequence mismatches between bovine or Xenopus and mouse sequences probably cause bovine and Xenopus to be clustered together. In the absence of this cross-species effect, if microarray hybridization were all performed on a same-species 
array, we would expect bovine and mouse to group closer together and Xenopus further apart. However, the fact that replicates cluster together by their appropriate species gives confidence in the reliability of the crossspecies hybridizations.

During the past few years, a number of studies have successfully used cross-species hybridizations while others have tried to assess their specificity and reliability in reflecting biological processes. Kane et al. (2000) showed that probe specificity requires target genes to be at least $75 \%$ similar over the target region when 50-mer oligonucleotide microarrays are used. In addition, if the target region is marginally similar (50-75\%), a stretch of complementary sequence of more than 15 contiguous bases will allow hybridization. Ji et al. (2004) reached fairly similar conclusions when they created a simple mathematical model for cross-species hybridization and concluded that a contiguous-matched oligonucleotide of $16 \mathrm{bp}$ was sufficient to generate a specific hybridization signal. In our previous study, nucleotide sequence similarity for genes expressed in the oocyte was evaluated between mouse, bovine, and $X$. laevis. We found that, on average, bovine sequences showed an $86 \%$ identity to mouse sequences while $80 \%$ identity was observed between Xenopus and mouse sequences (Vallee et al. 2006). Another group quantified the agreement in gene expression profiles across different species. Although the agreement was most robust when the target RNA was derived from closely related species $(<10$ million years divergence), consistent profiles for more distantly related species $(\sim 65$ million years divergence) and, to a lesser extent, even very distantly related species (>200 million years divergence) were also obtained (Renn et al. 2004). Finally, a recent study demonstrated that once the data are filtered by restricting proper cut-offs of probe homology, cross-species hybridization can closely reflect the biological process analyzed by same-species hybridization (Bar-Or et al. 2006). Although cross-species hybridizations have proven to be relatively specific, the identification of specific paralogues might prove to be difficult and need to be taken into consideration when results are analyzed. Occasional low sequence homology between common genes could preclude identification of the totality of transcripts conserved across species but, will not affect the validity of the genes identified as conserved in oocytes in the present study.

Moreover, the degree of homology between probes and targets when cross-species hybridizations are performed can be extremely variable. In the presence of sequence mismatches, relative hybridization intensities will reflect both differences in transcript abundance and differences in hybridization kinetics. In addition, intensities can be variable when two different species are used for cross-species hybridization, especially when the species studied are not equally divergent. In view of these limitations, the goal of this study was not to assess gene expression levels but rather to identify transcripts present in the oocytes of all three species. The microarray analysis revealed that a substantial number of genes are detected in bovine and Xenopus oocytes; 9853 and 10046 genes respectively. Previously, these labeling and hybridization protocols on this microarray platform and for all genes tested were extensively validated by quantitative real-time PCR; a log signal intensity $>2.0$ confirmed their expression (Carter et al. 2003). Thus, adjusting our threshold to above log 3.0 in the present study should be adequate. Moreover, a recently published study using the same microarray dataset also used a log-intensity signal $>3.0$ as the criterion for a positive signal (Mager et al. 2006). Finally, we previously showed through validation by RT-PCR and gene-specific microarray hybridization that crossspecies microarray hybridizations with oocytes from these three species were highly specific for the candidate genes tested (Vallee et al. 2006).

When we performed comparative analyses of genes expressed in mouse, bovine, and Xenopus, the results showed that $74 \%$ of genes expressed in mouse oocytes are common to the three species, providing evidence that most genes expressed in oocytes are conserved among species. This conforms with our previous study in which cross-species hybridizations on a cDNA multispecies microarray showed that $81 \%$ of the genes expressed in mouse oocytes were also expressed in bovine and Xenopus oocytes (Vallee et al. 2006). Another study compared the ESTs generated from a mouse oocyte cDNA library to genes expressed in $X$. laevis and Ciona intestinalis oocytes (Evsikov et al. 2006). This analysis revealed that for $80 \%$ of the genes expressed in the mouse oocytes, homologs transcribed in eggs of either $X$. laevis or $C$. intestinalis were found (Evsikov et al. 2006). Interestingly, a recent study on yeast has demonstrated that proteins with essential functions evolve more slowly than less essential proteins (Zhang \& He 2005). It concluded that this is likely to apply to mammalian genes as well. The authors suggest that when a large dataset of mouse knockout studies becomes available, it will be possible to demonstrate the same phenomena in mammals.

GO terms identified as being over-represented in oocyte-expressed genes conserved in all three species reflect the principal functions of the oocyte, such as RNA metabolism, ribonucleoprotein complex, RNA binding, cell cycle, and cellular division. It is well known that oocytes accumulate large quantities of maternal mRNAs during their growth phase (Rodman \& Bachvarova 1976) and that these maternal mRNAs are very stable with a half-life of 8-12 days (Brower et al. 1981). Thus, their timely recruitment for translation and/or degradation during early embryonic stages is critical for successful development. Genes expressed in the oocyte associated with cell cycle are critical for early embryonic development since maternal transcripts have to support 
cell cycles prior to embryonic genome activation, which occurs at different stages in different species (Telford et al. 1990). All these maternal factors, which are degraded following maturation (Piko \& Clegg 1982), are critical for the interval between fertilization and the maternalembryonic transition when transcriptional activity of the embryonic genome becomes fully functional. Pattern matching can be used to find genes with expression patterns similar to that of a selected template gene. In order to identify maternal genes, we used the spindlin (Spin) gene as template, a maternal transcript (Oh et al. 1997) that is known to be conserved in the three species (Vallee et al. 2006). Spin is known to be an abundant maternal transcript in the unfertilized egg and also in the 2-cell but not the 8-cell mouse embryo (Oh et al. 1997). SPIN protein is a substrate in the MOS/MAP kinase pathway that associates with the meiotic spindle and is suggested to play a role in cell-cycle regulation during the transition from gamete to embryo (Oh et al. 1997, 1998). Previously published microarray data on mouse preimplantation development (Hamatani et al. 2004) were used in this pattern-matching analysis to identify conserved maternally expressed genes. Expression profiles from these microarray data had previously been analyzed by a $k$-means nonhierarchical clustering method to identify expression trends in mouse embryonic development (Hamatani et al. 2004). The results of these studies showed that three different expression patterns corresponded to maternally expressed genes, and Spin was found in one of those clusters (Hamatani et al. 2004). Different studies have used microarray experiments to identify maternal transcripts in mouse oocytes (Hamatani et al. 2004, Wang et al. 2004, Zeng et al. 2004, Cui et al. 2007, Su et al. 2007), bovine oocytes (Misirlioglu et al. 2006, Adjaye et al. 2007, Fair et al. 2007), and in Xenopus (Evsikov et al. 2006, Graindorge et al. 2006), but to our knowledge, it has never been done for identifying conserved maternally expressed transcripts during development. The present patternmatching analysis allowed us to identify which of our conserved oocyte-expressed genes are expressed in mouse oocytes and degraded throughout embryonic development. We believe that genes identified in this analysis are inclined to be maternally expressed in bovine and $X$. laevis. This suggests that they have important functions in oogenesis, oocyte maturation, fertilization and/or early embryonic development and should therefore be further characterized.

In summary, the results obtained from this crossspecies hybridization allowed numerous conserved oocyte-expressed genes to be identified in mouse, bovine, and Xenopus, and also allowed conserved maternally expressed genes to be identified. Globally, this comparative analysis of oocyte transcript profiles revealed a high degree of conservation among species and clearly establishes the feasibility of working with a well-characterized microarray platform from another species. Since our understanding of the oocyte transcriptome and the identity of key oocyte-expressed genes are far from complete, the strategic use of cross-species microarray hybridizations - an original and nonstandard use of microarray - allowed the extraction of a smaller number of candidate genes that have important roles from a large cohort of maternal transcripts detected by microarrays. Finally, this study adds a substantive amount of new information that is extremely valuable to the scientific community working on fertility and early embryonic development. It will contribute to the elucidation of crucial molecular mechanisms and pathways regulating oogenesis and embryogenesis in different species including in humans, which may lead to the solutions for infertility problems or improvements in assisted reproductive technologies.

\section{Materials and Methods}

\section{Tissue collection}

Bovine ovaries were collected from a slaughterhouse. Cumulusoocyte complexes (COCs) from 3 to $6 \mathrm{~mm}$ follicles were collected by aspiration; germinal vesicle (GV) oocytes were mechanically denuded, washed several times in PBS to prevent cumulus cell contamination. Three groups of 200 GV oocytes were then frozen in a minimal volume of PBS in liquid nitrogen. $X$. laevis oocytes were obtained from an adult female anesthetized in $0.1 \%$ methanesulfonate salt of 3-aminobenzoic acid ethyl ester (MS222, Sigma-Aldrich) for $20 \mathrm{~min}$. A piece of ovary was isolated and oocytes were defolliculated for $1 \mathrm{~h}$ at $18{ }^{\circ} \mathrm{C}$ in OR2 saline containing $0.15 \%$ collagenase (Sigma). Stage IV-V oocytes $(\sim 1 \mathrm{~mm}$ diameter) were collected in OR2 medium at $18{ }^{\circ} \mathrm{C}$. Three groups of 20 oocytes were washed in PBS and frozen in liquid nitrogen until RNA extraction. Animals were cared for according to the respective recommended codes of practice and killed by an acceptable method approved by the local Animal Care Committee following the guidelines of the Canadian Council on Animal Care (1993).

\section{RNA extraction}

Bovine and Xenopus total RNA were extracted using the Absolutely RNA Microprep Kit (Stratagene) according to the manufacturer's instructions. An additional phenol/chloroform purification step was performed on Xenopus oocytes prior to total RNA extraction to remove excess lipids. The extracted RNA was dissolved in water and the integrity and concentration were evaluated using a 2100-Bioanalyzer (Agilent Technologies, Palo Alto, CA, USA) with the RNA PicoLab Chip (Agilent Technologies).

\section{Labeling and hybridization on the NIA 22 K 60-mer oligo microarray}

Three RNA aliquots (100 ng) for each species were labeled with Cy3-dye by two-round linear amplification labeling to make 
each cRNA target using a Fluorescent Linear Amplification Kit (Agilent Technologies) as previously described (Carter et al. 2003). In brief, mRNA was used to synthesize double-stranded cDNA with Moloney Murine Leukemia Virus (MMLV) reverse transcriptase in a reaction scaled down to a total volume of $4 \mu \mathrm{l}$, with half the standard T7-oligo-dT primer concentration and $125 \mathrm{ng} / \mu \mathrm{l}$ T4gp32 single-stranded DNA-binding protein (United States Biochemical, Cleveland, $\mathrm{OH}$, USA). Linear amplification was performed in a total volume of $16 \mu \mathrm{l}$, with half the standard NTP concentration and no labeled CTP. For the second round of amplification, the product of the first reaction was labeled using the manufacturer's standard protocol, with the addition of T4gp32 in the cDNA synthesis reaction. The quality and size distribution of targets were determined by RNA 6000 Nano Lab-on-chip Assay (Agilent Technologies) and quantified using a NanoDrop microscale spectrophotometer (NanoDrop, Wilmington, DE, USA). Similarly, universal mouse reference RNA (Stratagene), labeled with Cy5-dye by one round of linear amplification, was used as control in all hybridizations (Carter et al. 2003). cRNA targets (750 ng) from oocytes and universal reference were assembled into a hybridization reaction on the NIA Mouse 22K Microarray v1.0 (manufactured by Agilent Technologies; Carter et al. 2003). Three replicate cross-species hybridizations were performed for bovine and Xenopus for a total of six hybridizations and 121680 gene expression measurements. This microarray platform contains 60-mer oligonucleotide probes representing 20281 mouse transcripts, assembled primarily from sequences of stem cell, oocyte, and early embryo cDNA libraries. A complete list of the annotated gene content of the microarray can be found on the NIA mouse cDNA project website (http://lgsun.grc.nia.nih.gov/cDNA/).

\section{Microarray data analysis}

For our cross-species hybridizations, spot intensity was extracted from scanned microarray images using Feature Extraction 5.1.1 software (Agilent Technologies), which performs background subtractions and dye normalization as described previously (Carter et al. 2003). The microarray experiments presented in this study adhere to the standards proposed by the Microarray Gene Expression Data Society Microarray (www.mged.org). Data for the microarray experiments reported herein are stored in the public repositories ArrayExpress (E-MEXP-1339) (www.ebi.ac.uk/arrayexpress). Comparative analyses were performed using mouse preimplantation development microarray data from a study by Hamatani et al. (2004). Their results were obtained from the hybridization of mouse oocytes and embryos (unfertilized oocyte, fertilized oocyte, 2-cell, 4-cell, 8-cell, morula, and blastocyst) in four replicates on the NIA Mouse 22K Microarray v1.0. Microarray data from all three species were uploaded in the NIA Array Analysis tool (Sharov et al. 2005a) where background threshold was determined according to the plot of error function (S.D. (=square root of the error variance) versus expression level (Log intensity)). For each gene on the array, a mean log signal intensity was calculated on the basis of the signal intensities obtained from the three replicate hybridizations. Genes with a mean log signal intensity above the calculated threshold ( $>\log 3.0$ ) were considered as expressed. Correlation coefficients of signal intensities of all microarray spots between replicated experiments $(n=3$ for bovine and Xenopus, $n=4$ for mouse) were calculated in a pair-wise manner using the NIA Array Analysis tool (Sharov et al. 2005a). Furthermore, data processing including hierarchical clustering and pattern matching was also performed through the NIA microarray analysis tool. The pattern-matching algorithm can identify a group of genes, the expression patterns of which match to a selected template gene. The analysis was performed using microarray data from bovine and Xenopus oocyte and mouse preimplantation development microarray data from Hamatani et al. (2004) using the spindlin gene (Z00008605-1) as template, a tenfold change threshold and a 0.90 correlation threshold. The NIA Mouse Gene Index 8.0 (Sharov et al. 2005b) was used to find the GO terms over-represented among the conserved genes expressed in oocytes. This tool associates the proper GO terms from the Gene Ontology Consortium annotation categories for each gene and generates a list of over-represented GO terms in our population (Ashburner et al. 2000). The GO Consortium has created a defined vocabulary of terms describing the biological processes, cellular components, and molecular functions of all genes. It assigns genes to GO terms providing annotation and biological context for individual genes.

\section{Acknowledgements}

The authors acknowledge Drs Alexei A Sharov and Mark G Carter for their help in planning the experiment and analysis and Drs Susan Novak and Julie Fradette for critical reading of the manuscript and for providing valuable comments. MV is supported by NSERC fellowship. This research was supported in part by the Intramural Research Program of the NIH, the National Institute on Aging, the Canada Research Chair and Natural Science and Engineering Research Council of Canada. Lennoxville Dairy and Swine R \& D Center Contribution No. 936. The authors declare that there is no conflict of interest that would prejudice the impartiality of this scientific work.

\section{References}

Adams MD, Kelley JM, Gocayne JD, Dubnick M, Polymeropoulos MH, Xiao H, Merril CR, Wu A, Olde B, Moreno RF et al. 1991 Complementary DNA sequencing: expressed sequence tags and human genome project. Science 252 1651-1656.

Adjaye J, Herwig R, Herrmann D, Wruck W, Benkahla A, Brink TC, Nowak M, Carnwath JW, Hultschig C, Niemann H et al. 2004 Crossspecies hybridisation of human and bovine orthologous genes on high density CDNA microarrays. BMC Genomics 583.

Adjaye J, Herwig R, Brink TC, Herrmann D, Greber B, Sudheer S, Groth D, Carnwath JW, Lehrach H \& Niemann H 2007 Conserved molecular portraits of bovine and human blastocysts as a consequence of the transition from maternal to embryonic control of gene expression. Physiological Genomics 31 315-327.

Altmann CR, Bell E, Sczyrba A, Pun J, Bekiranov S, Gaasterland T \& Brivanlou AH 2001 Microarray-based analysis of early development in Xenopus laevis. Developmental Biology 236 64-75.

Arraztoa JA, Zhou J, Marcu D, Cheng C, Bonner R, Chen M, Xiang C, Brownstein M, Maisey K, Imarai M et al. 2005 Identification of genes expressed in primate primordial oocytes. Human Reproduction 20 476-483. 
Ashburner M, Ball CA, Blake JA, Botstein D, Butler H, Cherry JM, Davis AP, Dolinski K, Dwight SS, Eppig JT et al. 2000 Gene ontology: tool for the unification of biology. The Gene Ontology Consortium. Nature Genetics 25 25-29.

Bar-Or C, Bar-Eyal M, Gal T, Kapulnik Y, Czosnek H \& Koltai H 2006 Derivation of species-specific hybridization-like knowledge out of crossspecies hybridization results. BMC Genomics 7110.

Bermudez MG, Wells D, Malter H, Munne S, Cohen J \& Steuerwald NM 2004 Expression profiles of individual human oocytes using microarray technology. Reproductive Biomedicine Online 8 325-337.

Brower PT, Gizang E, Boreen SM \& Schultz RM 1981 Biochemical studies of mammalian oogenesis: synthesis and stability of various classes of RNA during growth of the mouse oocyte in vitro. Developmental Biology 86 373-383.

Burns KH, Viveiros MM, Ren Y, Wang P, DeMayo FJ, Frail DE, Eppig JJ \& Matzuk MM 2003 Roles of NPM2 in chromatin and nucleolar organization in oocytes and embryos. Science 300 633-636.

Canadian Council on Animal Care 1993 Guide to the Care and Use of Experimental Animals, Ottawa: Canadian Council on Animal Care.

Carter MG, Hamatani T, Sharov AA, Carmack CE, Qian Y, Aiba K, Ko NT, Dudekula DB, Brzoska PM, Hwang SS et al. 2003 In situ-synthesized novel microarray optimized for mouse stem cell and early developmental expression profiling. Genome Research 13 1011-1021.

Chalmers AD, Goldstone K, Smith JC, Gilchrist M, Amaya E \& Papalopulu N 2005 A Xenopus tropicalis oligonucleotide microarray works across species using RNA from Xenopus laevis. Mechanisms of Development 122 355-363.

Chismar JD, Mondala T, Fox HS, Roberts E, Langford D, Masliah E, Salomon DR \& Head SR 2002 Analysis of result variability from highdensity oligonucleotide arrays comparing same-species and crossspecies hybridizations. BioTechniques 33 516-518 (520, 522 passim).

Cui XS, Li XY, Yin XJ, Kong IK, Kang JJ \& Kim NH 2007 Maternal gene transcription in mouse oocytes: genes implicated in oocyte maturation and fertilization. Journal of Reproduction and Development 53 405-418.

Dalbies-Tran R \& Mermillod P 2003 Use of heterologous complementary DNA array screening to analyze bovine oocyte transcriptome and its evolution during in vitro maturation. Biology of Reproduction 68 252-261.

Diatchenko L, Lau YF, Campbell AP, Chenchik A, Moqadam F, Huang B, Lukyanov S, Lukyanov K, Gurskaya N, Sverdlov ED et al. 1996 Suppression subtractive hybridization: a method for generating differentially regulated or tissue-specific cDNA probes and libraries. PNAS 93 6025-6030.

Dobson AT, Raja R, Abeyta MJ, Taylor T, Shen S, Haqq C \& Pera RA 2004 The unique transcriptome through day 3 of human preimplantation development. Human Molecular Genetics 13 1461-1470.

Donaldson L, Vuocolo T, Gray C, Strandberg Y, Reverter A, McWilliam S, Wang Y, Byrne K \& Tellam R 2005 Construction and validation of a bovine innate immune microarray. BMC Genomics 6135.

Dong J, Albertini DF, Nishimori K, Kumar TR, Lu N \& Matzuk MM 1996 Growth differentiation factor-9 is required during early ovarian folliculogenesis. Nature 383 531-535.

Eberwine J 1996 Amplification of mRNA populations using aRNA generated from immobilized oligo(dT)-T7 primed cDNA. BioTechniques 20 584-591.

Evsikov AV, Graber JH, Brockman JM, Hampl A, Holbrook AE, Singh P, Eppig JJ, Solter D \& Knowles BB 2006 Cracking the egg: molecular dynamics and evolutionary aspects of the transition from the fully grown oocyte to embryo. Genes and Development 20 2713-2727.

Fair T, Carter F, Park S, Evans AC \& Lonergan P 2007 Global gene expression analysis during bovine oocyte in vitro maturation. Theriogenology $\mathbf{6 8}$ S91-S97.

Gasca S, Pellestor F, Assou S, Loup V, Anahory T, Dechaud H, De Vos J \& Hamamah S 2007 Identifying new human oocyte marker genes: a microarray approach. Reproductive Biomedicine Online 14 175-183.

Ghanem N, Holker M, Rings F, Jennen D, Tholen E, Sirard MA, Torner H, Kanitz W, Schellander K \& Tesfaye D 2007 Alterations in transcript abundance of bovine oocytes recovered at growth and dominance phases of the first follicular wave. BMC Developmental Biology 790

Graindorge A, Thuret R, Pollet N, Osborne HB \& Audic Y 2006 Identification of post-transcriptionally regulated Xenopus tropicalis maternal mRNAs by microarray. Nucleic Acids Research 34 986-995.
Grigoryev DN, Ma SF, Simon BA, Irizarry RA, Ye SQ \& Garcia JG 2005 In vitro identification and in silico utilization of interspecies sequence similarities using GeneChip technology. BMC Genomics 662.

Hamatani T, Carter MG, Sharov AA \& Ko MS 2004 Dynamics of global gene expression changes during mouse preimplantation development. Developmental Cell 6 117-131.

Ji W, Zhou W, Gregg K, Yu N \& Davis S 2004 A method for cross-species gene expression analysis with high-density oligonucleotide arrays. Nucleic Acids Research 32 e93.

Kane MD, Jatkoe TA, Stumpf CR, Lu J, Thomas JD \& Madore SJ 2000 Assessment of the sensitivity and specificity of oligonucleotide (50mer) microarrays. Nucleic Acids Research 28 4552-4557.

Ko MS, Kitchen JR, Wang X, Threat TA, Hasegawa A, Sun T, Grahovac MJ, Kargul GJ, Lim MK, Cui Y, et al. 2000 Large-scale cDNA analysis reveals phased gene expression patterns during preimplantation mouse development. Development 127 1737-1749.

Liang P \& Pardee AB 1992 Differential display of eukaryotic messenger RNA by means of the polymerase chain reaction. Science 257 967-971.

Mager J, Schultz RM, Brunk BP \& Bartolomei MS 2006 Identification of candidate maternal-effect genes through comparison of multiple microarray data sets. Mammalian Genome 17 941-949.

Misirlioglu M, Page GP, Sagirkaya H, Kaya A, Parrish JJ, First NL \& Memili E 2006 Dynamics of global transcriptome in bovine matured oocytes and preimplantation embryos. PNAS 103 18905-18910.

Monk M, Holding C \& Goto T 2001 Isolation of novel developmental genes from human germ cell, oocyte and embryo cDNA by differential display. Reproduction, Fertility, and Development 13 51-57.

Neilson L, Andalibi A, Kang D, Coutifaris C, Strauss JF III, Stanton JA \& Green DP 2000 Molecular phenotype of the human oocyte by PCRSAGE. Genomics 63 13-24.

Oh B, Hwang SY, Solter D \& Knowles BB 1997 Spindlin, a major maternal transcript expressed in the mouse during the transition from oocyte to embryo. Development 124 493-503.

Oh B, Hampl A, Eppig JJ, Solter D \& Knowles BB 1998 SPIN, a substrate in the MAP kinase pathway in mouse oocytes. Molecular Reproduction and Development 50 240-249.

Patel OV, Bettegowda A, Ireland JJ, Coussens PM, Lonergan P \& Smith GW 2007 Functional genomics studies of oocyte competence: evidence that reduced transcript abundance for follistatin is associated with poor developmental competence of bovine oocytes. Reproduction 133 95-106.

Pennetier S, Uzbekova S, Guyader-Joly C, Humblot P, Mermillod P \& Dalbies-Tran R 2005 Genes preferentially expressed in bovine oocytes revealed by subtractive and suppressive hybridization. Biology of Reproduction 73 713-720.

Piko L \& Clegg KB 1982 Quantitative changes in total RNA, total poly(A), and ribosomes in early mouse embryos. Developmental Biology $\mathbf{8 9}$ 362-378.

Rankin T, Familari M, Lee E, Ginsberg A, Dwyer N, Blanchette-Mackie J, Drago J, Westphal H \& Dean J 1996 Mice homozygous for an insertional mutation in the Zp3 gene lack a zona pellucida and are infertile. Development 122 2903-2910.

Rankin T, Talbot P, Lee E \& Dean J 1999 Abnormal zonae pellucidae in mice lacking ZP1 result in early embryonic loss. Development 126 3847-3855.

Rankin TL, O'Brien M, Lee E, Wigglesworth K, Eppig J \& Dean J 2001 Defective zonae pellucidae in Zp2-null mice disrupt folliculogenesis, fertility and development. Development 128 1119-1126.

Renn SC, Aubin-Horth N \& Hofmann HA 2004 Biologically meaningful expression profiling across species using heterologous hybridization to a cDNA microarray. BMC Genomics 542.

Robert C, Barnes FL, Hue I \& Sirard MA 2000 Subtractive hybridization used to identify mRNA associated with the maturation of bovine oocytes. Molecular Reproduction and Development 57 167-175.

Robert C, Hue I, McGraw S, Gagne D \& Sirard MA 2002 Quantification of cyclin B1 and p34(cdc2) in bovine cumulus-oocyte complexes and expression mapping of genes involved in the cell cycle by complementary DNA macroarrays. Biology of Reproduction 67 1456-1464.

Rodman TC \& Bachvarova R 1976 RNA synthesis in preovulatory mouse oocytes. Journal of Cell Biology 70 251-257.

Schena M, Shalon D, Davis RW \& Brown PO 1995 Quantitative monitoring of gene expression patterns with a complementary DNA microarray. Science 270 467-470. 
Shah G, Azizian M, Bruch D, Mehta R \& Kittur D 2004 Cross-species comparison of gene expression between human and porcine tissue, using single microarray platform-preliminary results. Clinical Transplantation $1876-80$

Sharov AA, Piao Y, Matoba R, Dudekula DB, Qian Y, VanBuren V, Falco G, Martin PR, Stagg CA, Bassey UC et al. 2003 Transcriptome analysis of mouse stem cells and early embryos. PLoS Biology 1 E74.

Sharov AA, Dudekula DB \& Ko MS 2005a A web-based tool for principal component and significance analysis of microarray data. Bioinformatics 21 2548-2549.

Sharov AA, Dudekula DB \& Ko MS 2005b Genome-wide assembly and analysis of alternative transcripts in mouse. Genome Research 15 748-754.

Soyal SM, Amleh A \& Dean J $2000 \mathrm{FIG} \alpha$, a germ cell-specific transcription factor required for ovarian follicle formation. Development 127 4645-4654.

Stanton JL \& Green DP 2001 A set of 840 mouse oocyte genes with wellmatched human homologues. Molecular Human Reproduction 7 521-543.

Stanton JL \& Green DP 2002 A set of 1542 mouse blastocyst and preblastocyst genes with well- matched human homologues. Molecular Human Reproduction 8 149-166.

Stanton JL, Macgregor AB \& Green DP 2003 Gene expression in the mouse preimplantation embryo. Reproduction 125 457-468.

Steuerwald NM, Bermudez MG, Wells D, Munne S \& Cohen J 2007 Maternal age-related differential global expression profiles observed in human oocytes. Reproductive Biomedicine Online 14 700-708.

Su YQ, Sugiura K, Woo Y, Wigglesworth K, Kamdar S, Affourtit J \& Eppig JJ 2007 Selective degradation of transcripts during meiotic maturation of mouse oocytes. Developmental Biology 302 104-117.

Telford NA, Watson AJ \& Schultz GA 1990 Transition from maternal to embryonic control in early mammalian development: a comparison of several species. Molecular Reproduction and Development 26 90-100.

Tong ZB, Gold L, Pfeifer KE, Dorward H, Lee E, Bondy CA, Dean J \& Nelson LM 2000 Mater, a maternal effect gene required for early embryonic development in mice. Nature Genetics 26 267-268.

Vallee M, Gravel C, Palin MF, Reghenas H, Stothard P, Wishart DS \& Sirard MA 2005 Identification of novel and known oocyte-specific genes using complementary DNA subtraction and microarray analysis in three different species. Biology of Reproduction 73 63-71.
Vallee M, Robert C, Methot S, Palin MF \& Sirard MA 2006 Crossspecies hybridizations on a multi-species cDNA microarray to identify evolutionarily conserved genes expressed in oocytes. BMC Genomics 7113.

Velculescu VE, Zhang L, Vogelstein B \& Kinzler KW 1995 Serial analysis of gene expression. Science $\mathbf{2 7 0} 484-487$.

Wang QT, Piotrowska K, Ciemerych MA, Milenkovic L, Scott MP, Davis RW \& Zernicka-Goetz M 2004 A genome-wide study of gene activity reveals developmental signaling pathways in the preimplantation mouse embryo. Developmental Cell 6 133-144.

Weil MR, Macatee T \& Garner HR 2002 Toward a universal standard: comparing two methods for standardizing spotted microarray data. BioTechniques 32 1310-1314.

Wu X, Viveiros MM, Eppig JJ, Bai Y, Fitzpatrick SL \& Matzuk MM 2003 Zygote arrest 1 (Zar1) is a novel maternal-effect gene critical for the oocyte-to-embryo transition. Nature Genetics 33 187-191.

Yan C, Wang P, DeMayo J, DeMayo FJ, Elvin JA, Carino C, Prasad SV, Skinner SS, Dunbar BS, Dube JL et al. 2001 Synergistic roles of bone morphogenetic protein 15 and growth differentiation factor 9 in ovarian function. Molecular Endocrinology 15 854-866.

Yao J, Ren X, Ireland JJ, Coussens PM, Smith TP \& Smith GW 2004 Generation of a bovine oocyte cDNA library and microarray: resources for identification of genes important for follicular development and early embryogenesis. Physiological Genomics 19 84-92.

Zeng F \& Schultz RM 2003 Gene expression in mouse oocytes and preimplantation embryos: use of suppression subtractive hybridization to identify oocyte- and embryo-specific genes. Biology of Reproduction 68 31-39.

Zeng F, Baldwin DA \& Schultz RM 2004 Transcript profiling during preimplantation mouse development. Developmental Biology 272 483-496.

Zhang J \& He X 2005 Significant impact of protein dispensability on the instantaneous rate of protein evolution. Molecular Biology and Evolution 22 1147-1155.

Received 30 July 2007

First decision 24 August 2007

Revised manuscript received 4 December 2007

Accepted 7 January 2008 